\title{
Postoperative Recurrent Paraspinal Fibromatosis after Resection of Cervical Meningioma and Review of Literature
}

\author{
Ankur Gupta ${ }^{1}$ Nafisa S. Batta ${ }^{1} \quad$ Vikas Batra $^{1}$ \\ ${ }^{1}$ Department of Radiology, Mahajan Imaging, Sports Injury Centre, \\ Vardhman Mahavir Medical College \& Safdarjung Hospital, Delhi, \\ India \\ Address for correspondence Ankur Gupta, MD Radiodiagnosis, \\ 16 HIG Kavita Nagar, Raipur, Chhattisgarh 492 001, India \\ (e-mail: Drguptaankur30@gmail.com).
}

Indian J Radiol Imaging 2021;31:514-518.

\begin{abstract}
The extra-abdominal deep fibromatoses also called as desmoid tumor (DT) are rare musculoaponeurotic, histologically benign tumoral soft tissue lesions which are locally aggressive with high rate of recurrence and no metastatic potential. Here, we describe a rare case of postoperative paraspinal fibromatosis in female child after aciurgy of intraspinal cervical meningioma. The lesion was incidentally detected in routine follow-up study and showed significant interval growth over a period of 1 year. Surgical resection was performed after histopathological confirmation; however, recurrence

Keywords

- cervical spine

- desmoid tumor

- fibromatosis

- meningioma

- sarcoma was observed. Hitherto only seven cases of postoperative paraspinal fibromatosis have been reported in the literature. To the best of authors' knowledge, this report is the first case of postoperative fibromatosis in cervical spine after resection of meningioma. The aim of the case report is to emphasize the clinical scenario for suspicion of fibromatosis, magnetic resonance imaging clues, and multidisciplinary treatment strategy which has shifted toward initial active surveillance.
\end{abstract}

\section{Introduction}

Desmoid tumor (DT) is a benign clonal fibroblastic proliferation that arises in the deep soft tissues and is classified as D48.1 in the International Classification of Diseases. This entity has definite female predilection. The cases of extra-abdominal DT are mostly sporadic and common sites are chest wall, shoulder girdle, thigh, and head and neck. Spinal presentation of fibromatosis is very rare. Magnetic resonance imaging (MRI) is the modality of choice for disease evaluation. It has characteristic histopathological-immunohistochemical profile, which helps arriving at final diagnosis. The multidisciplinary treatment approach is initial active surveillance, and later active treatment (resection, systemic therapy, and radiotherapy) if disease worsens.

published online July 27,2021
DOI https://doi.org/

10.1055/s-0041-1734357 ISSN 0971-3026

\section{Case Report}

A 14-year-old girl presented with posterior neck pain, paraesthesia, motor weakness, and gait disturbance for 12 months. MRI (1.5 Tesla GE healthcare Signa HDXT) of cervical spine was performed with axial, sagittal, and coronal images using T1-weighted (W) turbo spin echo (TSE), T2W TSE integrated with fat suppression and contrast-enhanced T1W sequence in multiple planes.

MRI revealed an intradural extramedullary mass at $\mathrm{C} 3$ to C4 level on left side, occupying more than $50 \%$ of the spinal canal with significant cord compression. The tumor was slightly hypointense on T1W images and darkly hypointense on T2W images relative to the spinal cord. The lesion showed mild enhancement with dural tail in postcontrast images
(C) 2021. Indian Radiological Association.

This is an open access article published by Thieme under the terms of the Creative Commons Attribution-NonDerivative-NonCommercial-License, permitting copying and reproduction so long as the original work is given appropriate credit. Contents may not be used for commercial purposes, or adapted, remixed, transformed or built upon. (https://creativecommons.org/licenses/by-nc-nd/4.0/).

Thieme Medical and Scientific Publishers Private Ltd. A-12, Second Floor, Sector -2, NOIDA -201301, India 


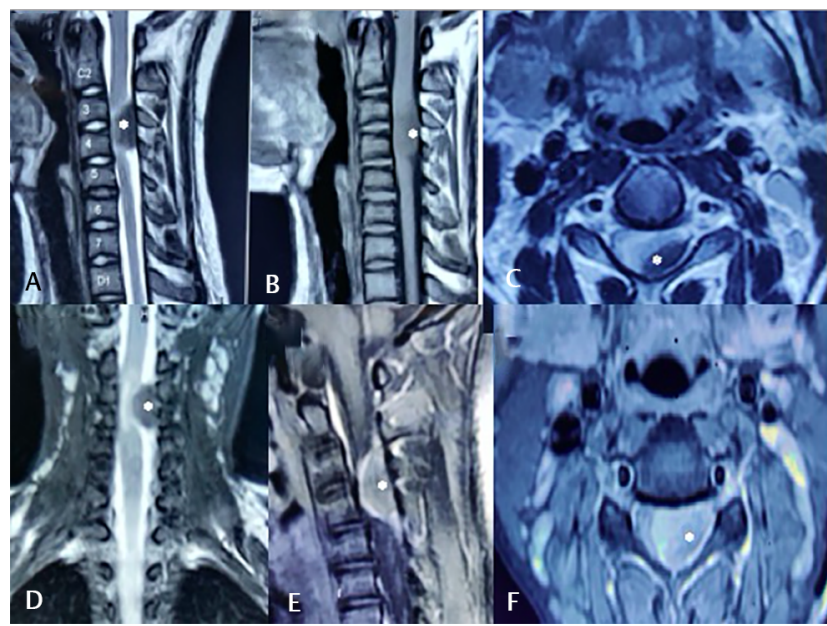

Fig. 1 (A-F) Contrast-enhanced magnetic resonance imaging reveals intradural extramedullary meningioma at $C 3$ to $C 4$ level compressing the spinal cord, in 14-year-old girl (white asterisk). Sagittal T2-weighted image (A), axial T2-weighted image (C), and coronal T2-weighted image (D) reveal darkly T2 hypointense lesion relative to spinal cord. Sagittal T1-weighted image (B) reveals iso- to hypointense signal of lesion relative to spinal cord. Sagittal postcontrast (E) and axial postcontrast (F) T1-weighted images reveal mildly enhancing lesion with dural tail.

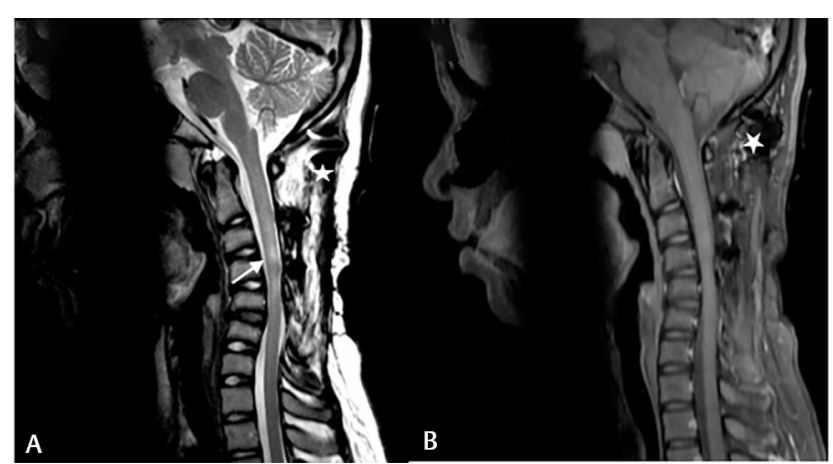

Fig. 2 (A, B) Contrast-enhanced magnetic resonance imaging 1 year after resection of intradural extramedullary cervical meningioma: Sagittal T2-weighted image $(\mathbf{A})$ reveals short segment myelomalacia from C3 to C5 level without any recurrent or residual intraspinal meningioma (white arrow). Sagittal postcontrast T1-weighted image (B) reveals no intraspinal enhancing lesion. The "white star" marked in both images reveals magnetic susceptibility artifacts from surgical hardware.

(-Fig. 1A-F). On the basis of earlier imaging features, diagnosis of fibrous meningioma was considered. The patient underwent uneventful complete tumoral resection and specimen histopathology report revealed World Health Organization (WHO) grade I transitional meningioma with MIB-1 labeling index of $\sim 4$ to $5 \%$. Postoperative recovery was satisfactory with resolution of motor and sensory neurological signs and symptoms.

This is an open access journal, and articles are distributed under the terms of the Creative Commons Attribution-NonCommercial-ShareAlike 4.0 License, which allows others to remix, tweak, and build upon the work noncommercially, as long as appropriate credit is given, and the new creations are licensed under the identical terms.

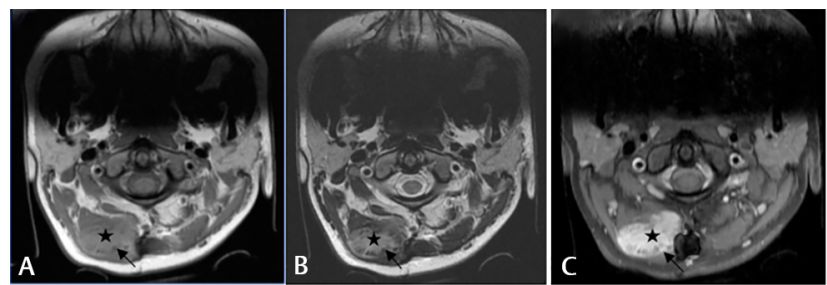

Fig. 3 (A-C) Contrast-enhanced magnetic resonance imaging 1 year after surgical resection of intradural extramedullary cervical meningioma: Axial T1-weighted (A) and axial T2-weighted (B) images depict incidentally detected ill-defined soft tissue lesion (black star) located near surgical hardware, centered in right paraspinal muscles from C1 to C3 level. The lesion is hypointense on T1-weighted and hyperintense on T2-weighted images. Peripheral low signal dark bands (black arrow), known as band sign, can be appreciated and is imaging clue to fibromatosis. Axial T1-weighted postcontrast image (C) reveals avid enhancement of lesion and nonenhancing low signal intensity bands within.

Contrast-enhanced MRI (CE-MRI) follow-up after 1 year revealed focal myelomalacia and mild cord thinning at the operative site without any residual or recurrent meningioma ( - Fig. 2A, B), and postoperative changes in the form of $\mathrm{C} 2$ to C6 laminectomy, laminoplasty, and magnetic susceptibility artifacts from miniplate and screws. An ill-defined new right paravertebral soft tissue lesion (not seen in preoperative MRI) measuring $2.9 \times 2.1 \mathrm{~cm}$ in axial and $4.9 \mathrm{~cm}$ in CC dimension, extending from $\mathrm{C} 1$ to $\mathrm{C} 3$ level, involving semispinalis capitis and cervicis muscles, and intermuscular fascial plane was seen ( - Fig. 3A-C). No obvious extension into spinal canal or intervertebral neural foramina was seen. The lesion exhibits hypointense signal on T1W images, intermediate to hyperintense signal on T2W images, and avid enhancement in postcontrast images. Band like nonenhancing low signals were appreciated at periphery (band sign). No evidence of gradient recalled echo blooming was seen.

On the basis of the earlier imaging findings and history of prior surgical instrumentation, deep fibromatosis was considered as first and foremost differential. Other less likely imaging possibilities considered were sarcoma, schwannoma, and myositis ossificans. Computed tomography-guided biopsy, and histopathological and immunohistochemical analysis were done for confirmation of the same, and it revealed typical conventional pattern of fibromatosis (spindle cells with indistinct borders arranged in long intersecting fascicles, showing moderate amount of eosinophilic cytoplasm and elongated nuclei with mild pleomorphism, in the background of collagenous matrix. No mitosis or necrosis is seen. On immunohistochemistry, tumor cells are positive for $\beta$-catenin, smooth muscle actin, S100, and epithelial membrane antigen, while are negative for desmin).

Since the patient was asymptomatic at this point of time, no active management was done and was subjected to active surveillance only, with recommended follow-up MRI after 3 months. The patient however came for follow-up after 9 months with clinically palpable lump, local pain, and restriction of neck movements. CE-MRI revealed significant interval growth in the size of right paravertebral lesion which now measured approximately $5.7 \times 7.0 \mathrm{~cm}$ in axial dimension 


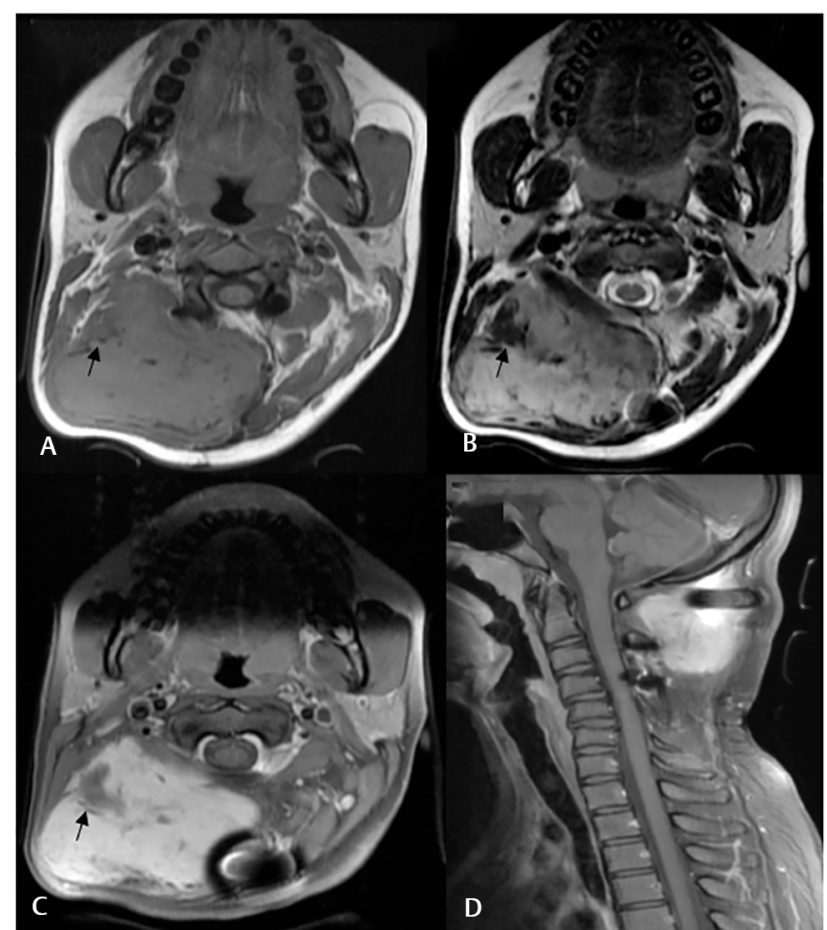

Fig. 4 (A-D) Contrast-enhanced magnetic resonance imaging after 9 months period of active surveillance: Axial T1-weighted (A), axial T2-weighted (B), axial T1 postcontrast (C), and sagittal T1 postcontrast (D) images reveal significant interval growth of desmoid tumor, seen near surgical hardware, now extending from $\mathrm{C} 1$ to C4 level. Imaging characteristics of the lesion essentially remain same ( $\mathrm{T} 1$ hypointense, $\mathrm{T} 2$ hyperintense, and avid postcontrast enhancement). Nonenhancing band like low signal intensities are now better seen (denoted by black arrow).

and $6.9 \mathrm{~cm}$ in $\mathrm{CC}$ dimension. The imaging characteristics of the lesion were essentially same as before, though now nonenhancing low signal intensity linear bands were better appreciated ( - Fig. 4A-D).

Surgical removal aiming at R0 resection was done. Gross examination of specimen revealed firm encapsulated tissue without hemorrhage or necrosis, and a nerve attached at periphery. Microscopic examination and immunohistochemical profile of specimen sections confirmed fibromatosis, with infiltrative margins focally.

Postoperative MRI after 6 months of interval was recommended; however, patient came for follow-up after 1 year with recurrent palpable lump. CE-MRI revealed a large recurrent, locally infiltrative similar morphology enhancing right paraspinal lesion measuring $8.0 \times 4.8 \mathrm{~cm}$ in axial dimension and $8.0 \mathrm{~cm}$ in CC dimension, now centered at different location, extending from C3 to D1 level. Typically, no hemorrhage or internal necrosis was demonstrated ( - Fig. 5A-D).

Presently, the case is in the tumor board-disease management group for further plan of action and is being considered for repeat surgical resection without or with local radiotherapy depending upon R0 or R1/R2 resection, respectively.

Review of literature reveals only seven cases of postsurgical paraspinal fibromatosis. This is a first case report of postoperative recurrent fibromatosis in cervical spine after the resection of meningioma ( $\mathbf{- T a b l e} \mathbf{1}$ ).

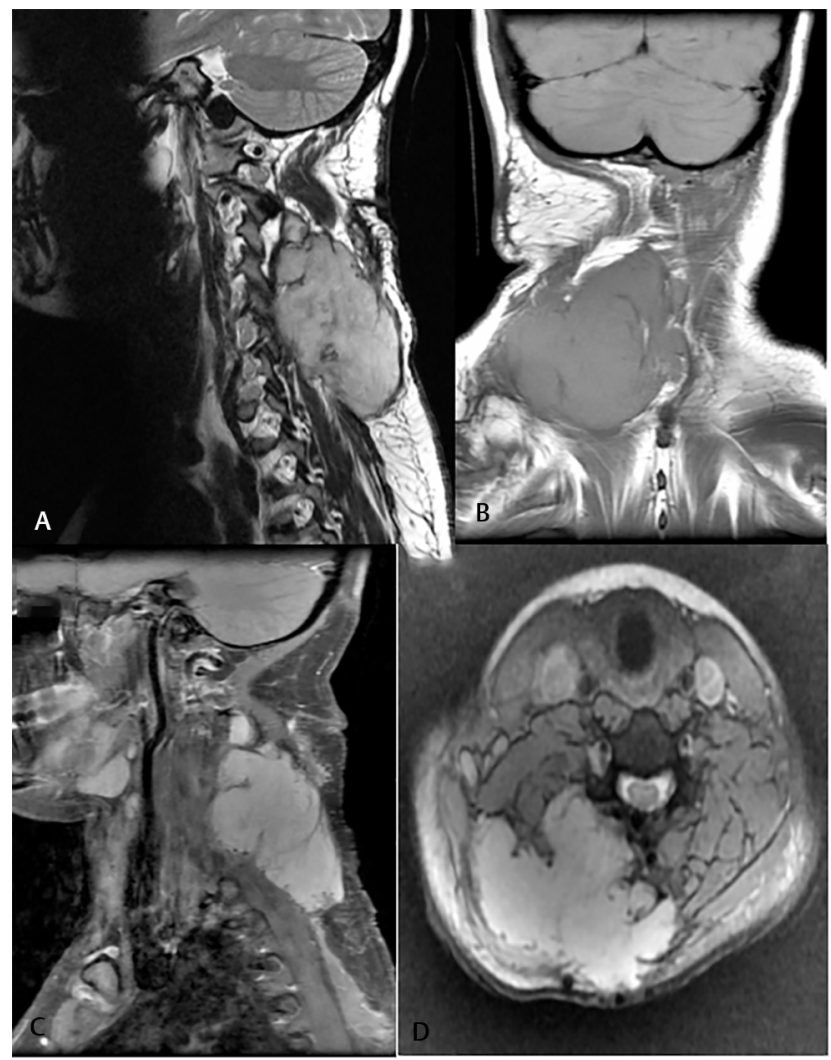

Fig. 5 (A-D) Contrast-enhanced magnetic resonance imaging 1 year after surgical resection of desmoid tumor: Sagittal T2-weighted (A), coronal T1-weighted (B), sagittal T1-weighted postcontrast (C), and axial gradient recalled echo (GRE) (D) images reveal large recurrent avidly enhancing tumor in right paraspinal region, now seen at different location, extending from C3 to D1 level. Imaging characteristics of the lesion is essentially same. Note is made of no internal necrosis, or GRE blooming to suggest hemorrhage or calcification.

\section{Discussion}

Fibromatosis is a benign lesion, which originates from proliferating fibroblasts and has variable and often unpredictable clinical course. It is categorized as superficial fibromatosis and deep fibromatosis. Superficial fibromatoses include palmar and plantar fibromatoses, Dupuytren's contracture, and Peyronie's disease. Deep fibromatosis (also synonymously called as DT, aggressive fibromatosis, and musculoaponeurotic fibromatosis), on the other hand, is often large, rapidly growing with high rate of recurrence, and tends to involve deep intramuscular anatomic compartments with frequent transspatial extension. ${ }^{1,2}$ According to the WHO, DT is a "clonal fibroblastic proliferation that arises in the deep soft tissues and is characterized by infiltrative growth and a tendency toward local recurrence but an inability to metastasize," even though it may be multifocal in the same limb or body part. ${ }^{2}$

Deep fibromatosis, depending on the anatomic site can be further classified as abdominal, intra-abdominal, and extra-abdominal. The abdominal musculoaponeurotic fibromatosis usually arises from the rectus or internal oblique muscles and fascia and intra-abdominal fibromatosis from mesentery, retroperitoneum, and pelvis, and together they 
Table 1 Summary of previously reported postoperative paraspinal fibromatosis

\begin{tabular}{|l|l|l|l|l|}
\hline Author & Age, sex & Spinal region & Spinal instrumentation & Tumor resection \\
\hline Gonatas (1961) & $45, \mathrm{~F}$ & Cervical & None & None \\
\hline Wyler and Harris (1973) & $39, \mathrm{~F}$ & C6-D2 & None & None \\
\hline Lynch et al (1999) & 49, F & D9-D11 & None & Thoracic meningioma \\
\hline Guzey (2006) & 50, F & L4-L5 & L4-L5 posterior fixation & None \\
\hline Sevak et al (2012) & 48, F & C6-D1 & C6-D3 posterior fixation & Schwannoma \\
\hline Puvanesarajah et al (2013) & 57, F & D4 & D2-D7 posterior fixation & Vertebral hemangioma \\
\hline Bohl (2019) & 56, F & C2-D4 & D4-pelvis & None \\
\hline Current study & 14, F & C1-D1 & C1-C4 posterior fixation & Cervical meningioma \\
\hline
\end{tabular}

Source: Bohl et al. ${ }^{14}$

account for $57 \%$ of the total cases. Extra-abdominal fibromatosis (43\%) commonly occurs in the shoulder girdle, chest wall, inguinal region, thigh, and head and neck. DTs are associated with a high rate of local recurrence; however, lack metastatic potential. ${ }^{3,4}$

DT accounts for less than 3\% of all soft tissue tumors and $\sim 0.03 \%$ of all neoplasms, affecting two to four people per million and has two to three times more female predilection. The involved age group ranges from 15 to 60 years; however, can occur in other age groups also. The tumors mostly present sporadically, however, can be manifestation hereditary familial adenomatous polyposis (FAP). The risk factors include surgical trauma, repeated irritation, pregnancy, oral contraceptive pills, and sex steroids. These tumors are histologically benign, however, can be locally aggressive and invasive with significantly high recurrence rates ranging from 24 to $77 \%$ depending on the patient's age, location of the tumor, and resection margins. ${ }^{2,5}$

In our case, the risk factor was surgical trauma due to the resection of cervical meningioma. The incidence of pediatric meningioma is as low as $2.19 \%$ of whole-brain tumors. The ratio of intracranial to intraspinal tumors in children is $\sim 20: 1$; thus, intraspinal meningioma in childhood is extremely rare. Review of literature reports less than 100 cases of pediatric spinal meningioma. Meningioma may recur, especially as a result of incomplete resection. Few long-term studies reveal recurrence rate ranging from 1.3 to $32 \%$ in both adult and children. ${ }^{6}$

The clinical presentation and symptoms burden of DT vary significantly depending on size and location. It can just be incidental finding without any symptoms to start with, detected on imaging done for another disease, just like our case, or presenting symptom can be painless swelling and lump, limping or other difficulty using arms, hands, legs, and feet, and compromised joint or muscle mobility. ${ }^{5}$ The intra-abdominal fibromatosis has entirely different presentation ranging from progressive abdominal pain to frank intestinal obstruction.

MRI is the modality of choice for evaluation of extra-abdominal DT; reveals poorly circumscribed, locally infiltrative, T2 intermediate to hyperintense, and T1 hypointense soft tissue lesion; and shows moderate to marked gadolinium-based contrast enhancement.
Nonenhancing low signal linear bands (band sign) are important imaging clue which can be seen in 62 to $91 \%$ of the cases, and pathologically represents collagenized hypocellular bands. Linear tails of extension along the aponeurosis are frequently seen in $~ 83 \%$ of cases, designated as "fascial tail sign." These DTs are usually centered in intramuscular location; hence, thin rim of surrounding fat can be seen, called as "split fat sign." The T2 signal intensity of the DT is directly proportional to its cellularity (i.e., higher T2 signal suggests higher fibroblast content). The response to systemic therapy and radiotherapy is depicted in MRI as decrease in T2 signal, enhancement, and size of lesion. ${ }^{4,7,8}$

The most common imaging differential is sarcoma. The sarcoma usually has pushing margins and may have necrosis, whereas fibromatosis usually has infiltrative margins and does not show necrosis; nevertheless, confirmative diagnosis requires tissue sampling and histopathological analysis. ${ }^{8,9}$ Other less likely imaging possibilities include neurogenic tumor, postoperative infection, hematoma, and reactive process such as myositis ossificans. ${ }^{3,5}$

The "conventional pattern" in microscopic examination is long sweeping fascicles with elongated, slender, spindle cells of uniform appearance, and pale cytoplasm set in collagenous stroma, parallelly arranged thin walled blood vessels and occasional sarcolemmic giant cells. The second most common pattern is hypocellular/hyalinized pattern.

The other less common patterns recognized are staghorn vessel pattern, myxoid pattern, keloidal pattern, nodular fasciitis-like pattern, and hypercellular pattern. Irrespective of the pattern, they unanimously show minimal cellular atypia and nuclear features lack hyperchromasia and any appreciable mitotic activity, that is, mitotic index $<1$. $^{10}$

The histologic differential diagnosis may vary depending on the anatomic location and includes low-grade sarcoma, organizing granulation tissue, nodular fasciitis, and inflammatory myofibroblast tumor. The immunohistochemical profile of fibromatosis typically described as positive staining for vimentin and for estrogen receptor- $\beta$, variable staining for smooth muscle actin, and nuclear positivity with $\beta$-catenin pinpoints the diagnosis. ${ }^{11,12}$

The APC gene $(5 \mathrm{q} 22.2)$ and $C T N N B l /$ catenin beta- 1 gene (3p22.1) mutation accounts for $89 \%$ of the cases of fibromatosis, both in sporadic and FAP associated, resulting in 
activation of canonical Wnt/B-catenin pathway. This leads to cytoplasmic upregulation of $\beta$-catenin, which then translocate to nucleus and interacts with transcriptional factors, ultimately resulting in myofibroblast proliferation and extracellular matrix (collagen) production. Genetic predisposition to DT in patients with FAP independent of germ line APC mutation has also been described, suggesting the existence of genes independent of APC that influence DT formation in FAP. ${ }^{2,13}$

Familial adenomatosis polyposis (especially in the Gardner variant of FAP) is a syndrome characterized by intestinal polyposis, osteomas, fibromas, and epidermal inclusion cysts. Patients with FAP are 1,000 times more prone to develop fibromatosis which can be multifocal and aggressive. It is more frequently associated with intra-abdominal desmoid-type fibromatosis, which develops in $\sim 5$ to $30 \%$ of the syndromic patients. ${ }^{2}$

The treatment of the DTs requires multidisciplinary approach. There is paradigm shift in management protocol in recent years. The frontline approach of the tumors at noncritical site, after diagnosis is "active surveillance" preferably on MRI for 1 to 2 years. It is a conservative approach which avoids over treatment in patients who are stable and pauci-symptomatic. In case of disease progression, active treatment is required (progression at a single imaging follow-up without significant increase in symptoms burden is not considered a green signal to start treatment, and wait for three subsequent progression if clinically possible is recommended). For the DT (such as mesenteric or head and neck DT) located in the vicinity of critical structure, involvement of which may result in significant morbidity or mortality, earlier decision toward an active treatment is considered. The active treatment is generally guided according to the anatomical site: For abdominal wall DT, surgery (R0 resection is goal) is the first option and for intra-abdominal DT (mesenteric, retroperitoneal, and pelvic) and extra-abdominal DT (extremity, girdles, chest wall) systemic therapy is the first treatment option, unless the expected morbidity by surgery is low. Further treatment options include continuation of medical therapy, local radiotherapy, or surgery. The recommended dose of radiotherapy is 50 to $60 \mathrm{~Gy}$, given in 1.8 to $2.0 \mathrm{~Gy}$ doses per exposure. The systemic therapy includes nonsteroidal anti-inflammatory drugs, antihormonal agents such as tamoxifen, and chemotherapy drugs viz. methotrexate plus vinblastine or vinorelbine, anthracycline-based regimens, and pegylated liposomal doxorubicin and tyrosine kinase inhibitors such as imatinib, nilotinib, sorafenib, and

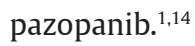

\section{Conclusion}

DTs/deep fibromatosis is albeit rare, but important consideration in patients (mostly female) presenting with new paraspinal soft tissue lesion in the region of prior spinal surgery or instrumentation. CE-MRI do play a key role in suggesting this entity (T2 hyperintensity, avid enhancement, band sign and fascial tail sign, and absence of internal necrosis and hemorrhage) and to ascertain the extent of disease. Sarcoma, schwannoma, and myositis ossificans (in postoperative scenario) are important imaging differentials which should not be forgotten, and hence for final confirmative diagnosis, microscopy and immunohistochemistry are required.

\section{Declaration of Patient Consent}

The authors certify that they have obtained all appropriate patient consent forms. In the form, the patient(s) has/have given his/her/their consent for his/her/their images and other clinical information to be reported in the journal. The patients understand that their names and initials will not be published and due efforts will be made to conceal their identity, but anonymity cannot be guaranteed.

\section{Financial Support and Sponsorship}

Nil.

\section{Conflicts of Interest}

None declared.

\section{References}

1 Desmoid Tumor Working Group. The management of desmoid tumours: a joint global consensus-based guideline approach for adult and paediatric patients. Eur J Cancer 2020;127:96-107

2 Skubitz KM. Biology and treatment of aggressive fibromatosis or desmoid tumor. Mayo Clin Proc 2017;92(6):947-964

3 Lacayo EA, Glastonbury CM, Hoang JK, Magliocca KR, Hill KL, Hudgins PA. Deep neck fibromatosis after diskectomy and cervical fusion: case series and review of the literature. AJR Am J Roentgenol 2016;206(5):1068-1072

4 Walker EA, Petscavage JM, Brian PL, Logie CI, Montini KM, Murphey MD. Imaging features of superficial and deep fibromatoses in the adult population. Sarcoma 2012;2012:215810

5 Goldstein A, Hoang S, Miller DC, Mesfin FB. Extra-abdominal desmoid tumor mimicking cervical spine schwannoma. Cureus 2018;10(8):e3145

6 Cho HR, Lee JK, Paik AL, Jang WY. An unusual cervical spinal meningioma in a child. J Korean Neurosurg Soc 2013;53(2):129-131

7 Sundaram M, McGuire MH, Schajowicz F. Soft-tissue masses: histologic basis for decreased signal (short T2) on T2-weighted MR images. AJR Am J Roentgenol 1987;148(6):1247-1250

8 Lee JC, Thomas JM, Phillips S, Fisher C, Moskovic E. Aggressive fibromatosis: MRI features with pathologic correlation. AJR Am J Roentgenol 2006;186(1):247-254

9 Peabody TD, Simon MA. Principles of staging of soft-tissue sarcomas. Clin Orthop Relat Res 1993;(289):19-31

10 Zreik RT, Fritchie KJ. Morphologic spectrum of desmoid-type fibromatosis. Am J Clin Pathol 2016;145(3):332-340

11 FletcherJA, Bridge JA, Hogendoorn PC, Mertens F, Desmoid-type fibromatoses. In: WHO Classification of Tumors of Soft Tissue and Bone. Lyon, France: IARC Press; 2013 72-73

12 Bhattacharya B, Dilworth HP, Iacobuzio-Donahue C, et al. Nuclear beta-catenin expression distinguishes deep fibromatosis from other benign and malignant fibroblastic and myofibroblastic lesions. Am J Surg Pathol 2005;29(5):653-659

13 Salas S, Chibon F, Noguchi T, et al. Molecular characterization by array comparative genomic hybridization and DNA sequencing of 194 desmoid tumors. Genes Chromosomes Cancer 2010;49(6):560-568

14 Bohl MA, Leveque JC, Bayles S, Sethi R. Postoperative development of desmoid tumor after surgical correction of adult spinal deformity: Case report and review of literature. World Neurosurg 2019;128:4-10 